View Article Online / Journal Homepage / Table of Contents for this issue MORRELL AND BELLARS: ACTION OF

\title{
XXXV.--Action of Hydrogen Peroxide on Carbohydrates in the Presence of Ferrous Sulphate. Part V.
}

\section{By Robert Selby Morrell and Albert Ernest Bellars.}

IN this communication, attempts have been made to trace the disappearance of different sugars during their oxidation by observing the diminution in the rotation angle, and from the determination of the initial and final reducing powers of the solutions, as well as their acidities, to obtain a fuller knowledge of the many oxidation stages which occur. The results of the change in the optical activity show that during successive additions of hydrogen peroxide up to 1 grammolecule for the same weight of carbohydrate the diminution in angle is proportional to the amount of oxidising agent added. The relative decrease in the angle depends on the sugar oxidised; galactose shows a greater diminution than glucose or fructose, maltose less than sucrose, which, in turn, has a smaller decrease in angle than lactose. In the case of arabinose, it would appear as if only 50 per cent. of the sugar were acted on by 1 gram-molecule of peroxide. Rhamnose, originally dextrorotatory, becomes levorotatory on oxidation, and the rotation is practically constant after 2 gram-atoms of oxygen have been added. This lævorotation appears to be due to the presence of a lævorotatory keto-acid. The high values of the final reducing powers must be due to the strong reducing powers of keto-acids and osor es formed in the oxidation. The acidities of the solutions after oxidation are not large, and are insufficient to account even for the complete formation of one monobasic hexose acid.

The smaller the yield of osazone precipitated by phenylhydrazine in the cold, the greater is the acidity of the solution. Attempts were made in the case of arabinose and rhamnose to isolate the acids formed during the oxidation. The simpler acids, formic and oxalic, were easily detected, but the more important keto-acids which were expected could not be isolated, although qualitative experiments seem to leave 
little doubt of their presence. Whether these contain the same or a smaller number of carbon atoms than the parent sugar it is impossible to say.

The method of E. Fischer and E. F. Armstrong (Ber., 1902, 35, 3141 ) has been applied to the formation of arabinosone from arabinose, rhamnosone from rhamnose, and, by using o-nitrobenzaldehyde, glucosazone and rhamnosazone may be made to yield the corresponding osones.

Autoxidation experiments have been performed with benzaldehyde as inductor, whereby the oxidations of glucose and fructose to glucosone in the presence of ferrous sulphate have been slightly accelerated, but the yields of osones were very poor.

Radium emanations were found to have no influence on the oxidation of carbohydrates. The small changes in optical activity were found to be due to alteration in concentration of the sugar solutions. When the conditions were arranged so that change in the concentration of the solution became impossible, the optical activity remained constant.

\section{ExPERIMENTAL.}

The Change in Optical Activity of Sugar Solutions during Oxidation by Hydrogen Peroxide in the Presence of Ferrous Sulphate.

Twenty per cent. sugar solutions containing 1 per cent. of crystallised ferrous sulphate were treated with 20 volume hydrogen peroxide, and, after the addition of each one-tenth of a gram-atom of oxygen, the optical activity of the solutions was observed. It was found in the case of the hexoses that the diminution in angle was nearly proportional to the amount of hydrogen peroxide added. Glucose, fructose, and galactose were the hexoses examined, and it will be seen from the curve (Fig. 1) that the decrease in angle of the two former sugars is practically the same, whereas for galactose the diminution in the optical activity is much greater. The changes in the activity of arabinose and rhamnose solutions were observed, and it was found that the curve for arabinose lies between that of glucose and galactose, whilst a similar curve for rhamnose shows a change from a dextrorotatory to a lævorotatory power.

In the bioses, the diminution in optical activity is evidently connected with the hydrolytic decomposition of the sugars with acids. Maltose, lactose, and sucrose are acted on according to their power of undergoing resolution into two hexoses.

In the case of glucose and fructose, the curve showing the diminution in angle lies nearly midway between the curve expressing the decrease in angle due to dilution only and that due to the disappear- 
ance of the sugar to yield inactive prodncts. This observation cannot be taken as strictly representing a disappearance of 50 per cent. of the sugar, for, although glucosore is feebly lævorotatory (E. Fischer, Ber., 1889, 22, 94), acids are formed during the oxidation, some of which may have an optical activity of the same sign as that of the parent sugar. In the case of galactose, the curve lies much closer to the dilution and loss of sugar curve than in the case of those

FIG. 1. *

Oxidation of Carbohydrates.

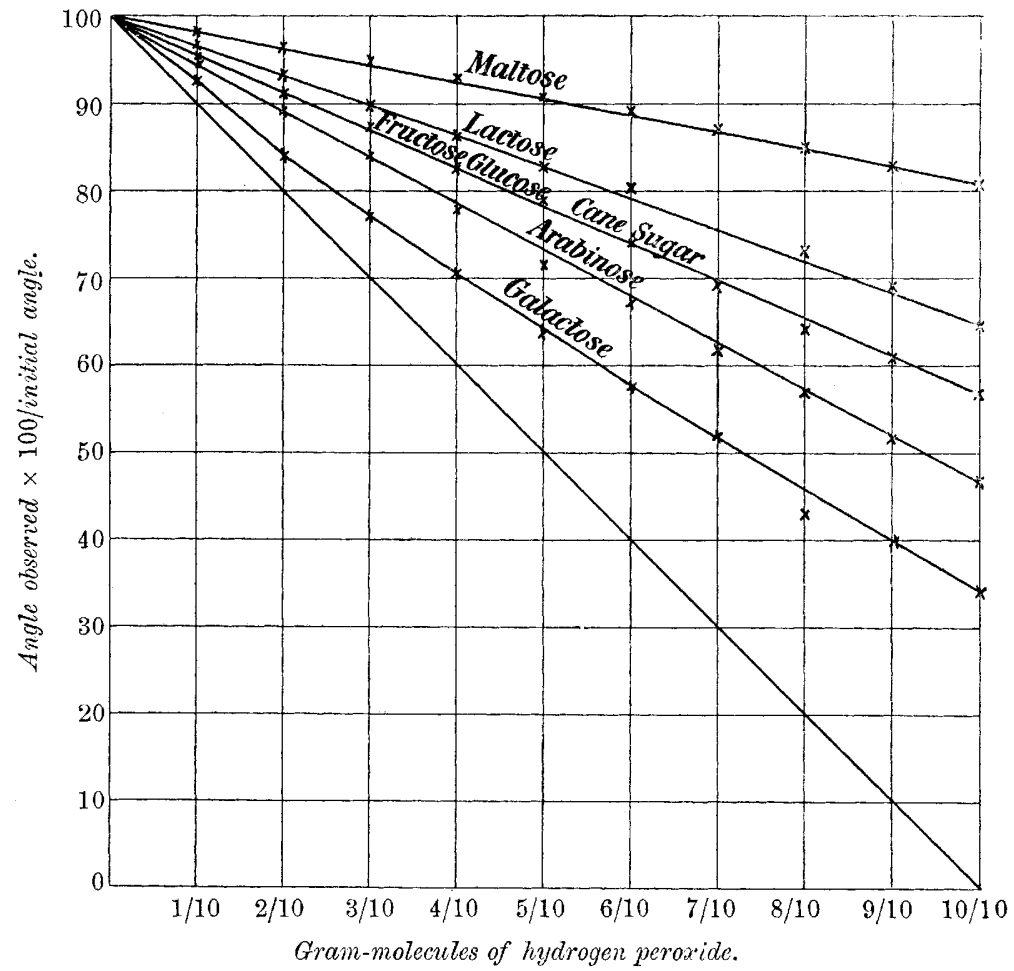

for glucose and fructose; moreover, the acidity at the end of the oxidation is much greater. It is unlikely that the corresponding hexose acids are formed in any quantity. Ruff (Ber., 1898, 32, 550) has shown that these acids on being heated are attacked in the presence of hydrogen peroxide and ferric salts to give aldoses containing one carbon atom less. Moreover, such aldoses in the presence of ferrous sulphate would undergo further oxidation to give (sones and acids

* Diminution in angle due to dilution has been eliminated. 
containing fewer carbon atoms (Morrell and Crofts, Trans, 1899, 75, 796). Keto-acids are more probable, since Ruff (Ber., 1898, 32, 2270) showed that in addition to $d$-arabinose, formic, glycollic, and a small quantity of a hexose acid, identified as Boutroux's oxygluconic acid, were obtained by the action of hydrogen peroxide on gluconic acid in the presence of ferric acetate.

A comparison of the curves for arabinose, namely, the calculated dilution curve, the curve representing the angular diminution during oxidation, and that due to dilution plus the disappearance of successive tenths of the amount of sugar up to ten-tenths, might indicate the disappearance of 50 per cent. of the sugar on the assumption that in addition to the osone only optically inactive acids are formed; glyceric acid is not produced, moreover lactic and tartaric acids have such small rotation angles compared with arabinose that their influence on the optical activity is negligible. It was thought that a further study of the oxidation of arabinose might furnish some clue as to the changes that occur during its oxidation, and at the same time might explain what took place in the case of other carbohydrates.

Ten grams of arabinose were dissolved in 50 c.c. of water, 0.5 gram of crystallised ferrous sulphate added, and 38.6 c.c. of hydrogen peroxide $(1$ c.c. $=0.0276$ gram 0 ) were introduced in one-tenths with the usual precautions. The action of the peroxide was very rapid, and in a few minutes the angle became constant, for example, at 3.15 p.m. 3.86 c.c. of hydrogen peroxide were run in, at $3.25 \mathrm{p} . \mathrm{m}$. the angle observed was $17.08^{\circ}$. At $5.15 \mathrm{p} . \mathrm{m}$. the angle was $16.89^{\circ}$, and 24 hours afterwards was $16.73^{\circ}$. The oxidation of carbohydrates is always very rapid, the hydrogen peroxide being used up as soon as it is added. Fig. 2 will show the relationship of the curve drawn from the observed data and those calculated for dilution, and for dilution plus disappearance of successive tenths of sugar to give inactive substances.

When the oxidation by one gram-molecule of peroxide had been completed, the acidity of the solution was determined. It was found to be 272 c.c. of $N / 10$ caustic soda, or 4080 c.c. of this solution for $1 \mathrm{gram}$-molecule of arabinose; to furnish $166 \mathrm{grams}$ of arabonic acid, 150 grams of arabinose would require 10 litres of $N / 10$ caustic soda. It was unlikely that the oxidation had proceeded only to the formation of arabonic acid, because the reducing power of the solution had diminished from 7452 to 5767 , a decrease of only one-fifth. The reducing power of the solution was estimated by means of Wood and Berry's method (Proc. Camb. Phil. Soc., 1903, 12, 98), whereby cuprous oxide is precipitated by a solution of copper potassium carbonate, and the washed cuprous oxide allowed to reduce a ferric sulphate solution; the ferrous sulphate formed was estimated by standard permanganate. The reducing power of the original sugar in the 
presence of ferrous sulphate was estimated previously, the amount of ferrous sulphate being in the same proportion as that used for the oxidation of the sugar by hydrogen peroside. The small diminution in the reducing power indicated the presence of residual sugar, of osone, and probably of a keto-acid containing 5 carbon atoms or less.

FIG. 2.

Oxidation of Arabinose.

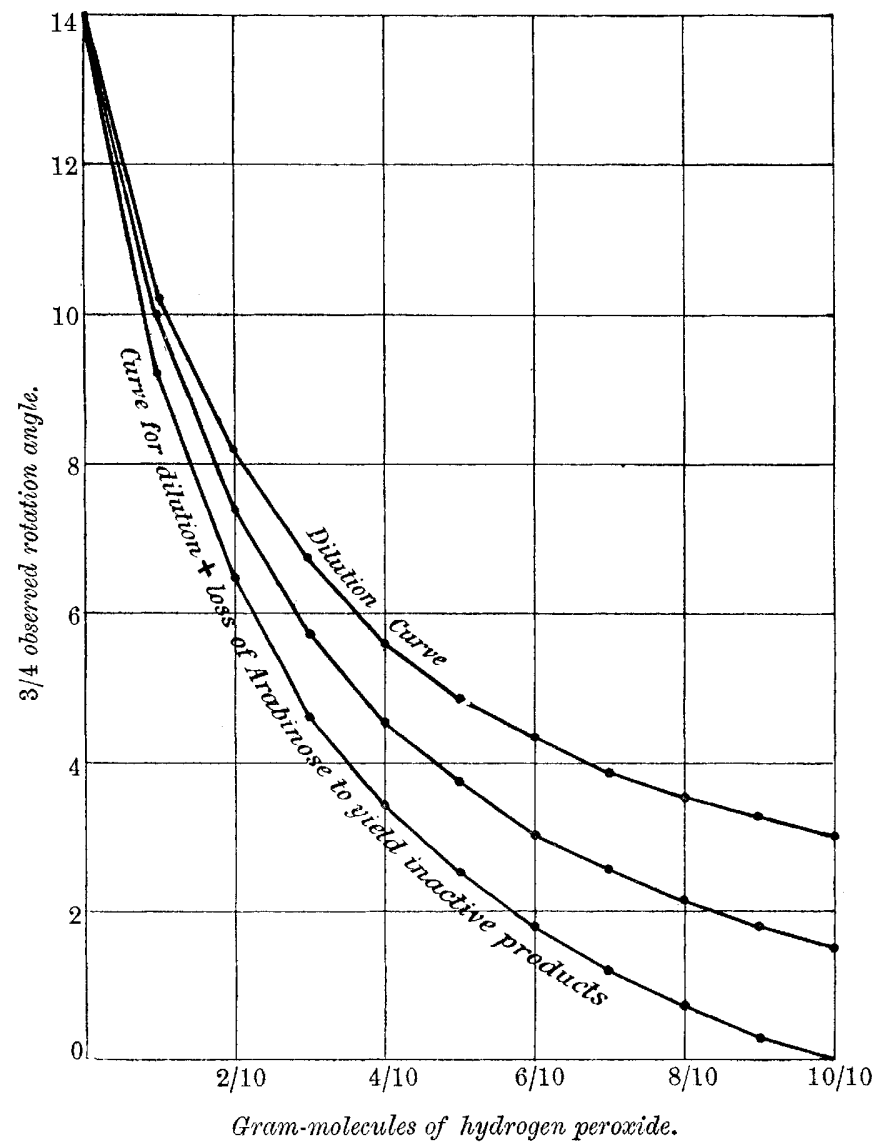

The yield of acids forming insoluble lead salts was small because normal lead acetate precipitated only $1 \mathrm{gram}$, and basic lead acetate only 3 grams of lead salt, but it is possible that these lead salts are soluble in excess of the precipitant, as was found to be the case with lead glycollate (Morrell and Crofts, Trans., 1903, 83, 1291). 
The yield of arabinosazone, after removal of the acids, was only 0.25 gram after the solution had remained for 6 hours at the ordinary temperature, but the yield was increased by 3 grams after heating the solution on the water-bath for 1 hour. Three grams of arabinosazone correspond, from experiments we have performed, with 5 grams of arabinose. The yield of arabinosone in this experiment was small, owing to the solution having remained alkaline for rather a long time during the filtration of the basic lead acetate precipitate, and E. Fischer has shown that osones are not stable in the presence of alkalis (Ber., $1889,22,94)$. In other experiments, we have found that by the oxidation of $10 \mathrm{grams}$ of arabinose, $1 \cdot 7 \mathrm{grams}$ of arabinosazone could be obtained at the ordinary temperature after one or two hours, and 1.7 grams of arabinosazone correspond, as will be shown later, to nearly 3 grams of arabinosone. An attempt was then made to prepare a small quantity of arabinosone by using E. Fischer's method (Ber., $1892,25,3141)$, and to determine its optical activity. Ten grams of recrystallised arabinosazone were added slowly to 1800 c.c. of boiling water containing 13 grams of benzaldehyde. After $2 \frac{1}{2}$ hours, the solution was filtered from unattacked arabinosazone and benzaldehydehydrazone, the filtrate shaken several times with ether, and concentrated to less than half its bulk in a vacuum at $50^{\circ}$. The liquid was again shaken several times with ether, decolorised with animal charcoal, and evaporated to a small volume. It was found to be slightly dextrorotatory, and gave an immediate precipitate at the ordinary temperature with phenylhydrazine acetate. In order to obtain a rough value of the specific rotation of the arabinosone, the syrup was evaporated to a constant weight in a platinum basin at the ordinary temperature under reduced pressure, whereby 0.2753 gram was obtained. This amount, when dissolved in 8 c.c. of water, gavo a rotation of $+0 \cdot 245^{\circ}$, whence $[a]_{\mathrm{D}}=7 \cdot 12^{\circ}$. The contents of the polarimeter tube were warmed with the calculated quantity of phenylhydrazine acetate for $1 \frac{1}{2}$ hours on the water-bath, the yield of arabinosazone precipitated was a little more than $0.1 \mathrm{gram}$. Since $[a]_{\mathrm{D}}$ for arabinose is $94.85^{\circ}$, the activity of the osone is very small compared with that of the sugar. With regard to the formation of a keto-acid from arabinose, the amounts of lead salts produced are so insignificant that further investigations would entail the use of larger quantities of the expensive carbohydrate. From 100 grams of arabinose, the yield of lead salts was so poor that their investigation was restricted to qualitative tests. The basic lead acetate precipitate was suspended in water and decomposed by hydrogen sulphide; the filtrate from lead sulphide was concentrated in a vacuum at $60^{\circ}$ and the distillate was neutralised with calcium carbonate, a small quantity of a crystalline calcium salt obtained, which reduced mercuric chloride to mercury, but 
neither reduced a Fehling's solution, nor reacted with phenylhydrazine acetate. With ferric chloride, it gave the reaction for an acetate, and on heating with dilute sulphuric acid the odours of acetic and formic acids were noticed. The residue in the distilling flask was boiled with calcium carbonate until neutral, filtered, evaporated to a small bulk, poured into alcobol, and filtered. The precipitated calcium salt reduced Fehling's solution readily, and reacted after slightly warming with phenylhydrazine to give a dark red precipitate. With $p$ bromophenylhydrazine, an uncrystallisable oil was obtained. Lime water added to a solution of the calcium salt gave a white precipitate, and normal lead acetate a white precipitate, but the calcium salt did not react with aniline oxalate to give the test for glyoxylic acid.

It is advisable to give tables showing the acidities, yields of osazones, and changes in reducing power of a series of sugars, before considering the attempts to identify the acids formed from rhamnose.

One gram-mol. of sugar + one gram-mol. of peroxide.

Glucose

Fruetose

Galactose

Maltose

Lactose

Sucrose

Arabinose

Khamnose
Acidity

expressed

in e.c.

of $N / 10$

$\mathrm{NaOH}$.

3600

3240

5778

(hydrazo-hy'drazide)

very small

practically none

51 grams

$25 \cdot 5$,

32,
Relucing power

in c.c. of

permanganate.

$\begin{array}{ccc}\text { Before. } & \text { After. } \\ 8600 & 6408 \\ 9854 & & 8971 \\ 7861 & 6152 \\ & \\ 8550 & 7251 \\ 8550 & 8225 \\ 7 & 8766 \\ 7452 & 5767 \\ 7500 & 5125\end{array}$

Diminution in reducing power. Per cent. $25 \cdot 5$

9

22

15

4

23

32

The yields of osazones given include that due to the osone and to the keto-acid which may have escaped precipitation by basic lead acetate. In the case of arabinose and rhamnose, the yields of osazones are larger if substituted hydrazines, such as phenylmethylhydrazine and $p$-bromophenylhydrazine are employed. The foregoing table shows that the greater the acidity in a series, the greater the diminution in angle of rotation. The reducing powers are not generally diminished more than 25 per cent., varying from 9 per cent. in the case of fructose to 32 per cent. in the case of rhamnose. It will be noticed that the reducing power of a cane sugar solution is almost that of a fructose solution, which is in agreement with the view that hydrolysis ensues before oxidation. The slight diminution in the reducing power indicated the formation of substances containing aldehyde and ketone groupings. Maltose and lactose, yielding small quantities of osones, have high acidities, due to the further oxidation of the osones, and high reducing powers owing to the presence of relatively large quantities of unattacked sugar. It is to be noticed that galactose furnishes the highest acidity number and a 
reducing power, after oxidation, relatively greater than glucose, although no osone is formed, but rather a keto-hexose acid. Mr. Crofts has informed us that the dark red substance precipitated from a cold solution of oxidised galactose by phenylhydrazine contained a substance which, after repeated crystallisation from benzene, melted at $152-155^{\circ}$, with decomposition, and gave on analysis the following numbers.

0.1120 gave 14 c.c. moist nitrogen at $12^{\circ}$ and $760 \mathrm{~mm} . \quad \mathrm{N}=14.9$.

$0.1450 \Rightarrow 0.308 \mathrm{CO}_{2}$ and $0.0785 \mathrm{H}_{2} \mathrm{O} . \mathrm{C}=58.0 ; \mathrm{H}=6.0$.

$\mathrm{C}_{18} \mathrm{H}_{32} \mathrm{O}_{5} \mathrm{~N}_{4}$ requires $\mathrm{C}=57 \cdot 7 ; \mathrm{H}=5 \cdot 9 ; \mathrm{N}=14.9$ per cent.

FIG. 3.

Oxidation of Rhamnose.

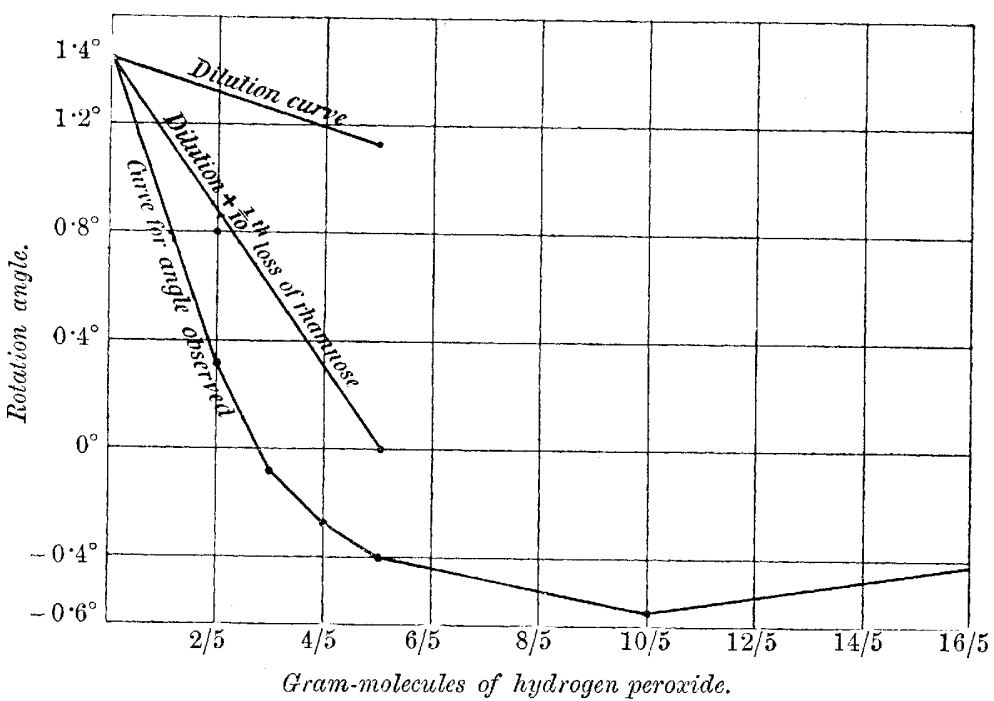

The substance, which is of a yellow colour, was deposited from benzene in nodular aggregates.

The curve for rhamnose $\left([a]_{\mathrm{D}}+7 \cdot 35^{\circ}\right)$ shown in Fig. 3, after the addition of 1 gram-atom of oxygen, is that for a lævorotatory solution. The angle diminished in one experiment from $+1.47^{\circ}$ to $-0.4^{\circ}$ for an addition of 1 gram-atom of oxygen. If the amount of the oxidising agent is increased up to 4 atoms, the fall in angle is very slow, attaining a maximum at 3 atoms of $-0.6^{\circ}$, and rising to $-0.4^{\circ}$ after 4 gram-atoms of oxygen have been added. After the addition of 2 gram-atoms of oxygen, the osone was found to be present in very small quantities, and the lævorotatory power must be due to 
the presence of an acid which might be rhamnonic acid. The disappearance of the osone after the addition of 2 gram-atoms of oxygen would point to the lævorotatory power being due to an acid or acids formed from the osone. The first acid formed from the osone would be a keto-acid, which would undergo further oxidation to yield two acids, oxalic or glyoxylic acid and a monobasic dihydroxyacid; this in turn would be oxidised further, although in what way it is impossible to predict. The formation of simpler acids, the activity of which is small, from the lævorctatory keto-acid will account for the small angular diminution consequent on the addition of more than 1 gram-atom of oxygen. Reference to the table on page 286 shows that the acidity is greater than that of an arabinose solution and the diminution in reducing power is more decided. A good deal of attention has been paid to rhamnose, since the properties of this sugar have been examined more fully, and the peculiar change in sign of the rotation angle merited some consideration. In one oxidation, where 1 gram-atom of oxygen was used, it was shown that this change of sign was due to an acid, precipitated by basic lead acetate, leaving the filtrate slightly dextrorotatory. The basic lead salt, on decomposition, yielded a lævorotatory solution, which reacted slowly with phenylhydrazine to give a dark red precipitate. The amount of lead salt formed was so small that purification was impossible, and it was thought that by employing larger quantities of the oxidising agent the weight of the basic lexd acetate precipitate might be increased. Two and four atoms of oxygen were used and more workable quantities of the basic lead salts were obtained. After removal of the insoluble lead salts, the solution was found to be slightly lævorotatory, and where four gramatoms of oxygen were used the filtrate was inactive, a fact which points to the complete disappearance of the original sugar. The normal lead acetate precipitate was found to consist chiefly of lead oxalate and sulphate. These were transformed into their calcium salts. The calcium sulphate was removed by washing with water and acetic acid, and analysed; the calcium oxalate left was reduced by sodium amalgam to glyoxylic acid, which was confirmed by the Hopkins reaction with tryptophan (Proc. Roy. Soc., 1901, 68, 21). Moreover, a calcium determination and estimation by standard permanganate confirmed the presencs of the calcium oxalate. From the basic lead acetate precipitate, when four atoms of oxygen were used, a calcium salt was obtained which contained $\mathrm{Ca}=18.87$ and 18.91 per cent.; it reduced Fehling's solution and reacted readily with substituted hydrazines, such as phenylhydrazine and $p$-bromophenylhydrazine, but the products did not admit of recrystallisation from solvents; moreover, the salt was not calcium glyoxylate, although it answered 
all the tests for glyoxylic acid except Hopkins's reaction and the aniline test. With two atoms of oxygen, the normal lead acetate precipitate yields in addition to oxalic acid a calcium salt which is partially soluble in alcohol, and the insoluble and soluble portions contain nearly the same percentage of calcium, namely, 16.87 and 17.14. It is impossible to assign with any confidence a formula to these salts, because their purity cannot be guaranteed, but it is of some interest that both calcium salts prepared in the same way react with phenylhydrazine acetate readily, and with increasing amount of hydrogen peroxide the calcium percentage is increased. Until more characteristic reactions of ketohydroxy-acids are discovered, the complete identification of acid products of the oxidation of the sugars will continue to be exceedingly difficult.

\section{Other Methiods of Preparing Osones.}

As in the preparation of arabinosone by Fischer and E. F. Armstrong's method (loc. cit.) fairly large quantities of rhamnosone can be prepared from rhamnosazone by the action of benzaldehyde. In one experiment, 5 grams of rhamnosazone were added slowly to 1 litre of boiling water containing 5 grams of benzaldehyde, the latter being kept in solution by the previous addition of 120 c.c. of absolute alcohol. After boiling for an hour, the benzaldehydehydrazone and unattacked rhamnosazone were filtered off, and the filtrate treated in the manner described under arabinose. From the concentrated solution, nearly 0.9 gram of rhamnosazone (m. p. $178^{\circ}$ ) was obtained by treatment with phenylhydrazine acetate. It was thought that if an aldehyde were used which yielded a hydrazone more insoluble in alcohol than benzaldehydehydrazone, the formation of an osone would proceed even in absolute alcoholic solution. $o$-Nitrobenzaldehyde was employed and it was found that 1 gram of the scarlet $o$-nitrobenzaldehydehydrazone was soluble in 50 c.c. of 80 per cent. hot alcohol, and crystallised on cooling in scarlet needles Five grams of rhamnosazone were dissolved in 100 c.c. of 90 per cent. alcohol, 8 grams of $o$-nitrobenzaldehyde and 150 c.c. of hot water added successively, and the liquid was heated for some time on the waterbath. On cooling, the osazone and unattacked o-nitrobenzaldebyde separated out; it seemed as if very little action had taken place. between the two substances. At $125^{\circ}$, the two substances reacted; 5 grams of rhamnosazone dissolved in 100 c.c. of 50 per cent. alcohol and heated with 8 grams of $o$-nitrobenzaldehyde in a sealed tube for 2 hours, gave a quantity of osone which yielded 0.3 gram of rhamnosazone (m. p. $178^{\circ}$ ). If the temperature were raised to $140^{\circ}$ and the heating prolonged to 8 hours, no osone could be detected. 
When 4.5 grams of glucosazone were heated with 11.5 grams of o-nitrobenzaldehyde in a 55 per cent. alcoholic solution at $100^{\circ}$ in a sealed tube, no $o$-nitrobenzaldehydehydrazone was formed; at $125^{\circ}$ the reaction commenced, and the scarlet hydrazone appeared. The contents of the tube were diluted with water, and the unattacked glucosazone and $o$-nitrobenzaldehyde were filtered off. The last traces of $o$-nitrobenzaldehyde were removed by ether, and the aqueous solution, after concentration in a vacuum, reacted wath phenylhydrazine at the ordinary temperature to give glucosazone (m. p. $\left.205^{\circ}\right)$. The yield of regenerated glucosazone was 17 per cent. of the original weight of glucosazone.

\section{Autoxidation Experiments with Benzaldehyde.}

Instead of using hydrogen peroxide as oxidiser, oxygen might be rendered active in the presence of an inductor, such as benzaldehyde. Engler and Wild (Ber., 1897, 30, 1669) state that the interaction of oxygen and benzaldehyde gives benzoyl peroxide and hydrogen peroxide. If the aldehyde alone be exposed to the oxygen, the product will be benzoic acid. Bödlander (Ahrens' Sammlung, 3, 470) assumed that benzoyl hydrogen peroxide, $\mathrm{C}_{6} \mathrm{H}_{5} \cdot \mathrm{CO} \cdot \mathrm{O} \cdot \mathrm{OH}$, was formed, which acted as a powerful oxidising agent. It was thought that it would be interesting to investigate whether benzaldehyde would accelerate the oxidation of the sugars in the presence of ferrous sulphate.

A stream of oxygen or air was drawn slowly through a fructose solution containing benzaldehyde and traces of ferrous sulphate in suspension. The oxidation of the iron was very rapid; after a few hours, the ferrous iron reappeared, disappearing when more air was passed in. The benzaldehyde gradually darkened in colour and became pasty, but even after several days it was not completely oxidised. After 4 days' contact with oxygen, the benzaldehyde was carefully removed by ether, and the solution was freed from ether by warming to $40^{\circ}$ in a vacuum. Addition of phenylhydrazine acetate gave an immediate turbidity and separation of glucosazone, which in $3 \frac{1}{2}$ hours at the ordinary temperature amounted to $0.4 \mathrm{gram}$ from 10 grams of fructose and melted on recrystallisation at $202-203^{\circ}$ (yield, 0.2 gram). A blank experiment with 2 grams of fructose in 20 c.c. of water, a drop of ferric chloride, and sodium acetate, tested with phenylhydrazine acetate, gave during the same time an exceedingly slight precipitate. In experiments conducted with glucose, it was found that the oxidation was very much slower than in the case of fructose, although when the stream of oxygen was cut off ferrous iron reappeared, which was immediately oxidised by bubbling in more oxygen. After 4 days, the benzaldehyde was removed by ether and 
the solution tested for glucosone by means of phenylhydrazine acetate under exactly the same conditions as in the case of fructose. The yield of glucosazone from 15 grams of glucose was 0.2 gram (m. p. $204^{\circ}$ ), less than one-third of what was furnished by fructose. Galactose was found to be oxidised slightly under the same conditions. The benzaldehyde during the oxidation changed to a semi-solid, brown mass, as if some of the ferric iron had been absorbed into the mixture of benzaldehyde and benzoic acid. In the presence of manganese sulphate, no change could be observed.

It was noticed that on bubbling air for 3 hours through a solution of fructose containing ferrous sulphate and a few drops of glacial acetic acid the sugar had been slightly oxidised. The solution reacted with phenylhydrazine acetate at the ordinary temperature after a quarter of an hour, but the yield of osazone was very small indeed. Fructose is invariably more easily attacked by active oxygen than glucose.

From fructose, in the presence of manganese sulphate, we were unable to obtain a solution which reacted with phenylhydrazine acetate at the ordinary temperature within 3 hours. If manganese acetate was present and oxygen was bubbled in, a decided darkening was noticed which slowly disappeared, but the oxidation was apparently very slow. The addition of alum had no effect on the rate of oxidation. The experiments were carried out in diffused daylight only.

\section{Experiments with Rudium Bromide as Catalytic Agent.}

Experiments on the action of radium salts were performed to find out whether the $\beta$. and $\gamma$ rays from radium bromide would accelerate the oxidation of fructose by air in the presence of ferrous sulphate. Unfortunately, the results are up to the present negative, but, nevertheless, a somewhat curious effect was obtained in the course of the work. It was thought that the change would be exceedingly small and difficult to measure. From the curves given in the earlier part of the paper, it is evident that the diminution in angle is proportional to the amourt of oxidising agent present. The experiments performed consisted in exposing a 20 per cent. aqueous solution of fructose, containing 1 per cent. of crystallised ferrous sulphate and 0.03 per cent. of mercuric chloride, in a thin glass dish or ebonite vessel with a mica bottom to the action of the $\beta$-and $\gamma$-rays from 5 milligrams and 20 milligrams of radium bromide. In order to prevent evaporation, the dish resting on the capsule containing the radium bromide was placed in the dark in a large bell-jar containing a vessel filled with water to which a trace of mercuric chloride had been added.

An exactly similar solution was placed in another bell-jar and kept 
in the dark and at the same temperature to serve as a blank experiment. In a second series of experiments, the sugar solutions were kept in a bell-jar which contained 5 per cent. sodium chloride solution. In a third series, the radium capsule was placed over the sugar solution. The solutions were examined in an "inversion" tube, whereby a constant temperature was secured by the water-jacket of the observation tube. The polarimeter employed was a triple field apparatus, which could be read accurately to $0.02^{\circ}$. In a fourth series of experiments, a glass tube containing 5 milligrams of radium bromide was left in contact with a sugar solution, containing ferrous sulphate, in a sealed flask filled with oxygen and kept in the dark for several weeks. In all cases, a similar blank experiment was carried out under exactly the same conditions. In the first series of experiments, the solution over radium gained in weight, whilst the blank solution lost slightly; this loss must have been due to evaporation during the weighing of the dish. The results were independent of the nature of the vessel, and were the same whether glass or ebonite vessels were employed. In six days, 13.7495 grams of a 20 per cent. fructose solution gained 0.0695 grams, whilst the blank solution lost 0.015 gram (the solutions were weighed every day). The solution over radium showed a slight diminution in optical activity, and was less by $0.25^{\circ}$ than the angle observed in the blank solution. After three weeks, the angle had diminished by $0.7^{\circ}$ and was $0.47^{\circ}$ less than that shown by the blank experiment. The two solutions possessed the same acidity, and showed no difference in their behaviour towards phenylhydrazine acetate ; in neither case was there an immediate precipitate of glucosazone.

In the second series of experiments, it was found that the solution in a glass vessel over which 20 milligrams of radium were placed lost weight faster than the blank solution, and the angle increased slightly in consequence. After 3 weeks, the angles differed only by $0.48^{\circ}$.

In the fourth series of experiments, after 5 days there was absolutely no change in the angles observed in the solution in the flask containing 5 milligrams of radium bromide, and in the blank experiment, even after 3 weeks' exposure, there was no change in the angle. The results may be summarised as follows: oxygen does not alter the rotation of a fructose solution in the presence of ferrous sulphate when the solution is kept in a large flask in the dark even after 5 days (compare Fenton and Jackson, Trans., 1899, 85, 10), and it does not alter the rotation of a similar fructose solution under the same conditions in the presence of a tube containing 5 milligrams of radium bromide even after 3 weeks. The changes in weight and small differences in angles observed must be due to alterations in the concentra- 
tions of the solutions. Probably the emanations from the radium bromide, by setting up convection currents, accelerate the evaporation from the water to the sugar solution in the first series of experiments, and from the sugar to the salt in the second series. When the radium capsule in the bell-jar was insulated from the surrounding air by means of ebonite and thick glass, the differences in the weight of the two solutions were much diminished, and there was, after 10 days, no alteration of optical activity in the two cases. The salt solution used had a greater osmotic pressure than the fructose solution, and the presence of a trace of mercuric chloride precluded any intervention of ferments. These experiments were brought to an abrupt conclusion by the 20 milligrams of radium bromide in the capsule absorbing water through the cover and losing their radioactivity.

The authors desire to acknowledge gratefully the receipt of a Government Grant from the Royal Society with the aid of which this investigation has been carried out.

Gonville and Caius College, CAMBRIDGE. 